This document is the accepted manuscript version of the following article:

Graf, M., Emmenegger, L., \& Tuzson, B. (2018). Compact, circular, and optically stable multipass cel1 for

mobile laser absorption spectroscopy. Optics Letters, 43(11), 2434.

http://doi .org/10.1364/oL.43.002434

\title{
Compact, circular, and optically stable multipass cell for mobile laser absorption spectroscopy
}

\author{
Manuel GraF ${ }^{1}$, Lukas Emmenegger ${ }^{1}$, and Béla Tuzson ${ }^{1}$ \\ ${ }^{1}$ Empa, Laboratory for Air Pollution \& Environmental Technology, CH-8600 Dübendorf, Switzerland \\ *Corresponding author: bela.tuzson@empa.ch \\ Compiled May 10, 2018
}

Compact and lightweight laser absorption spectrometers for accurate trace gas measurements are of great scientific and commercial importance. In these instruments, the multipass cell (MPC) represents a critical element in terms of achievable size and sensitivity. Herein, we introduce a versatile MPC-concept that unifies compactness, mechanical rigidity, and optical stability. Relying on fundamental cavity design principles and modern diamond turning techniques, we have developed a segmented circular MPC that allows efficient and interference-free beam folding. A prototype cell is presented featuring up to $10 \mathrm{~m}$ optical path length at a total mass of less than $200 \mathrm{~g}$. Incorporated in a highly compact setup without additional beam pre-shaping optics, we demonstrate a normalized noise level of low $10^{-4}(2 \sigma)$ at $1 \mathrm{~Hz}$.

OCIS codes: (220.0220) Optical design and fabrication; (280.3420) Laser sensors; (300.6360) Spectroscopy, laser; (120.6200) Spectrometers and spectroscopic instrumentation

https://doi.org/10.1364/OL.43.002434

Non-destructive and contact-free gas analysis by laser absorption spectroscopy is a powerful and widely applied measurement technique [1]. Nowadays, we face an increasing demand for compact and lightweight spectrometers to be deployed e.g. aboard of cars, UAVs, and balloons, or as part of planetary exploration missions [2-5]. Such mobile settings are of growing importance in a multitude of disciplines as they offer enhanced spatial and temporal sampling flexibility. With the advent of quantum cascade lasers (QCL), interband cascade lasers (ICL), and thermoelectrically cooled MCT-detectors, compact and portable mid-infrared sensors became feasible. However, high-precision measurements of molecular compounds require the extension of the optical path length (OPL) to improve the signal-to-noise ratio. In direct absorption spectroscopy, this is usually achieved by the incorporation of a multipass cell (MPC). Currently, this key element is the major limiting factor towards miniaturization, because the established designs do not satisfyingly combine both compactness and interference-free beam folding. To date, best documented precision and most efficient beam folding are reached in astigmatic Herriott cells [6] or variations thereof. However, these solutions are very sensitive to the relative posi- tioning of the mirrors, so that a considerable mechanical effort is required to realize and maintain their alignment. Moreover, the beam waist size and location must be accurately adjusted by additional optical elements to ensure low-noise operation. As a result, corresponding optical setups are bulky and heavy, limiting their applicability on portable platforms. Therefore, the design of suitable MPCs for mobile instruments has to be fundamentally reconsidered.

Depending on the specific target gas and deployment conditions, the requirements on an MPC can be very diverse. Nevertheless, common criteria for mobile in-situ spectrometers can be summarized as follows: (i) For compactness, a long OPL is desired at low overall mass and size. As these properties are mutually exclusive, the volume-normalized optical path length $\delta=\mathrm{OPL} / V$ is an important figure of merit. (ii) Low effective volumes are advantageous for high temporal sampling resolution. (iii) Mobile systems require mechanically robust cells to preserve their functionality upon thermally or mechanically induced stress. This can be achieved by either enhanced rigidity or by tolerance against misalignment. (iv) In order to benefit from the larger absorption signal, interference related optical noise must be suppressed. Therefore, it is crucial that the laser beam remains well defined upon multiple reflections. (v) In case of reactive or adhesive molecules, the exposed cell surface area should be kept low in order to reduce chemical interaction and memory effects. (vi) Finally, parasitic biases due to ambient air absorption can be avoided by minimizing the OPL outside the sampling volume.

In this Letter, we introduce a novel MPC-concept that takes into account all of the above criteria. We describe its design principles and present benchmark experiments acquired with a first prototype cell.

Recently, Tuzson et al. [7] presented the toroidal MPC as a lightweight alternative to collinear, two-mirror based cells or circular arrangements of individual mirrors [8]. The emergence of an $N$-star polygram when coupling a laser beam under an oblique angle to the inner surface of a cylindrical or toroidal surface, was shown to be a versatile optical path extension concept. Indeed, the monolithic circular geometry results in a mechanically robust and lightweight optical element, having optimal properties for open-path measurements and high flow rate applications. However, according to basic resonator theory, the inherently concentric mirror configuration in the radial plane of any toroidal surface inevitably leads to a divergence of laser 

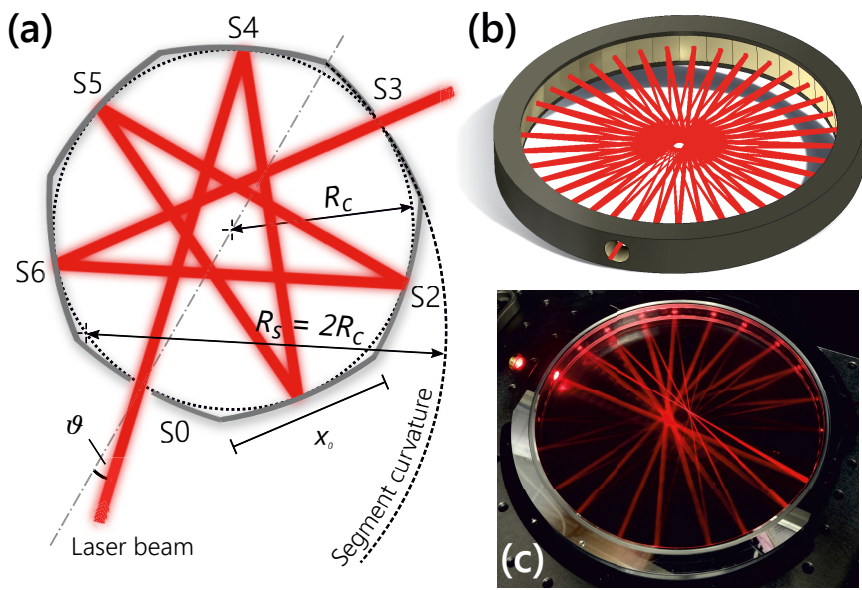

Fig. 1. (a) Schematic drawing of the inner surface of an SCMPC, here with 7 confocal segments. The coupling angle $\vartheta$ of the beam defines the reflection pattern by the rules of an $N$ star polygram. (b) Concept model and ray trace simulation. (c) Photograph of the cell prototype. The beam folding principle is visualized by a red trace laser and the alternating beam envelope can easily be observed. The angle $\vartheta$ was selected to realize 22 reflections, that is, a reduction of the maximally supported OPL to one third.

beams upon multiple reflections [9]. Hence, the outgoing radiation consists of a superposition of various beam components, causing strong optical fringes in the signal. As a workaround, the integration of an aperture mask has been proposed that constrains the reflective inner surface and thereby truncates the divergent radiation at each reflection $[7,10]$. While this measure leads to a reduction of the optical fringes, the successive generation of diffraction patterns at each aperture yields a significant complication of the design, optimization, and alignment process [11]. In the concept proposed here, we combine the mechanical advantages of the circular symmetry with inherent optical stability.

Within the framework of paraxial resonator theory, the criterion to confine light between equally curved concave mirrors in a stable manner can be derived as $R>L / 2$, i.e., the radius of curvature $R$ of the mirrors must be greater than half their separation distance $L$ [9]. It is obvious that this criterion cannot be fulfilled by any object that obeys continuous rotational symmetry. Thus, the only possibility to incorporate the above requirement into a circular geometry is to renounce the central axis of rotation and thereby breaking the continuous symmetry. This leads to a segmentation of the reflective inner surface [see Fig. 1(a)], where each segment is allowed to have a larger radius than the cell itself. As shown in Figs. 1(b, c), the emerging segmented circular multipass cell (SC-MPC) successfully prevents beam-divergence. The beam spot radius $w_{i}$ on each mirror segment $i$ can be calculated for given $R$ and $L$ [12]. To avoid spillover and beam-branching, the size of the mirror segments has to be dimensioned according to the largest occurring beam spot. Thus, we may define the full side length of the quadratic segments as $x_{0}=2 C \max \left(w_{i}\right)$, while the scaling factor $C$ should be chosen grater than $\pi$ to prevent leakage. Among all mirror arrangements fulfilling the stability criterion, the confocal arrangement $(R=L)$ is especially suitable for this application, since it supports the smallest beam spots $\left(w_{i, \text { conf }}^{2}=L \lambda / \pi\right)$. Therefore,
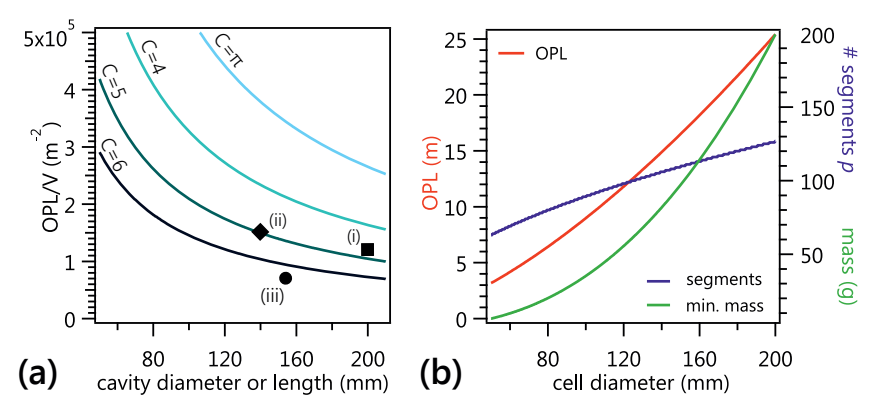

Fig. 2. (a) Calculated beam folding efficiency as function of the cell diameter for different segment scaling factors. Literature values from existing solutions are added as reference: i) astigmatic Herriott [6], ii) mask-enhanced toroidal [10], iii) herein presented prototype. (b) The maximal OPL (left scale), the minimal mass and the number of reflections (both right scale) are shown as a function of the cell diameter for $C=4$ and $\lambda=6 \mu \mathrm{m}$. The mass calculation assumes quadratic segments and a full aluminum body with wall strength-to-radius ratio of $1: 9$.

provided that the beam is pre-shaped to match the lowest-order Gaussian eigenmode, the cell reaches maximal compactness. For this case, we can derive the theoretical dependence of the cell diameter $d$ to its supported OPL. Under the assumption of small incoupling angles $\vartheta$ (i.e. dense patterns), which legitimates the approximation $d \simeq L=d \cos \vartheta$, the OPL is given by

$$
\mathrm{OPL}=p \cdot d=\frac{(d \pi)^{3 / 2}}{2 C \sqrt{\lambda}}
$$

with $\lambda$ being the wavelength of the laser and $p$ denoting the number of segments. According to this equation, the maximal OPL, minimal mass, and the corresponding number of segments are shown in Fig. 2(b) as a function of the cell diameter for a range of realistic examples. Figure 2(a) highlights the outstanding potential of the SC-MPC towards miniaturization. For small cell diameters, the beam folding efficiency is enhanced due to the small beam spot radii in such cavities. The comparison shows that the compactness of our current prototype (see below) is already close to state-of-the-art MPCs, even though the segment size was designed very conservatively.

A larger segments size, i.e. selection of a higher C-factor, yields increased tolerance against misaligned, imperfect or misshaped beams - at the expense of a somewhat larger cell diameter. Especially when coupling arbitrarily focused beams, the segment dimensions must account for larger and variable beam spot sizes. In a perfectly confocal arrangement, however, only two alternating beam shapes occur, which remain constant for any number of reflections (cf. Fig. 1(c)). Therefore, a confocal SC-MPC accepts any beam, whose spots completely fit onto two consecutive segments - regardless of the exact waist size and position. This is a key advantage because tedious pre-cell beam shaping becomes obsolete. In essence, collimated laser sources, as commercially available, can be directly attached to the cell. This possibility not only enables highly compact setups, but also increases the stability and avoids an optical path outside the sampling volume.

We designed and developed a prototype cell in order to validate the performance of the proposed concept in a spectroscopic application. The SC-MPC consists of a monolithic aluminum- 


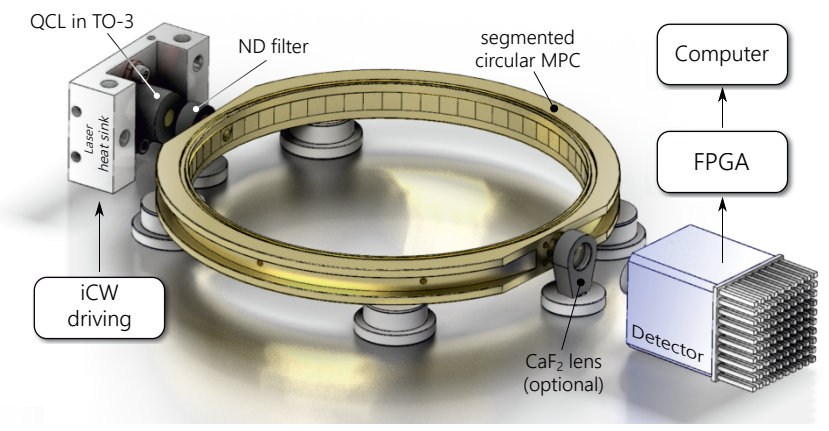

Fig. 3. Highly compact setup for spectroscopic benchmark experiments and noise characterization. Besides laser, SC-MPC, and detector, the setup features only two additional optical elements: a focusing lens and an ND filter. Alternatively, an individually curved last segment allows direct focusing onto the detector to gain further compactness of the system.

ring with inner radius $R_{c}=77.25 \mathrm{~mm}$. The segmented inner surface has been realized by single-point diamond turning (LT Ultra-Precision Technology $\mathrm{GmbH}$, Germany). Thereby, the servo-controlled lathe tool is periodically modulated in coordination with the cell revolving around its main axis. Such machining requires two-axis flexibility (axial and radial), potentially resulting in increased surface roughness compared to simpler geometries. The corresponding effects are discussed in more detail below. Sixty-five quadratic, spherically curved segments were shaped into the ring, each with a $7.47 \mathrm{~mm}$ side length and a radius of curvature $R_{S}=2 R_{c}=154.5 \mathrm{~mm}$. The width and height of the segments is chosen to offer appropriate alignment tolerance ( $C=6.9$ ). To enhance the surface quality, the segments are machined into a $0.2 \mathrm{~mm}$ thick chemically deposited nickel-phosphor layer. A galvanic gold plating (Epner Technology, U.S.) provides high mid-infrared reflectivity.

The in- and output apertures are placed at opposite sides (segments 0 and 33), providing a maximum of 64 passes. This configuration has been selected to ensure enough space for optical components to be placed directly at the cell. The laser beam enters under an angle $\vartheta=\frac{\pi}{2}(1-2 q / p)$, with $q$ denoting the pattern-density. By selecting $q$ close to $p / 2$, we minimize the creation of an astigmatic beam upon reflection and ensure a highdensity pattern. Thus, a maximal OPL of $9.89 \mathrm{~m}$ is reached for $q=\lfloor p / 2\rfloor=32$ resulting in $\vartheta=1.38^{\circ}$. By variation of $\vartheta$, a different first segment can be selected, leading to a reduction of the OPL, e.g. by a factor $1 / 3$, as shown in Fig. 1(c). For low-pressure measurements, the cell can be closed with two anodized aluminum lids and tilted $\mathrm{CaF}_{2}$-windows at the apertures. Thereby, the effective sampling volume becomes $140 \mathrm{ml}$, implying a beam folding efficiency of $\delta=0.7 \times 10^{5} \mathrm{~m}^{-2}$. The mass of the cell itself amounts to $198 \mathrm{~g}$.

Benchmark experiments were performed by direct laser absorption spectroscopy using a cw-DFB-QCL (Alpes Lasers, Switzerland) emitting at $7.83 \mu \mathrm{m}$. The laser is packaged in a TO3-housing with built-in collimation-lens. By placing the laser directly at the input aperture of the cell, the OPL outside the sampling volume is drastically reduced. Due to the high optical power up to $30 \mathrm{~mW}$, a neutral density filter $(\mathrm{ND}, \mathrm{OD}=1.0)$ was inserted between the laser and cell to prevent detector saturation. The special curvature of the last segment $\left(R_{S 64}=320 \mathrm{~mm}\right)$ enables in-cell beam focusing onto a directly attached detector.
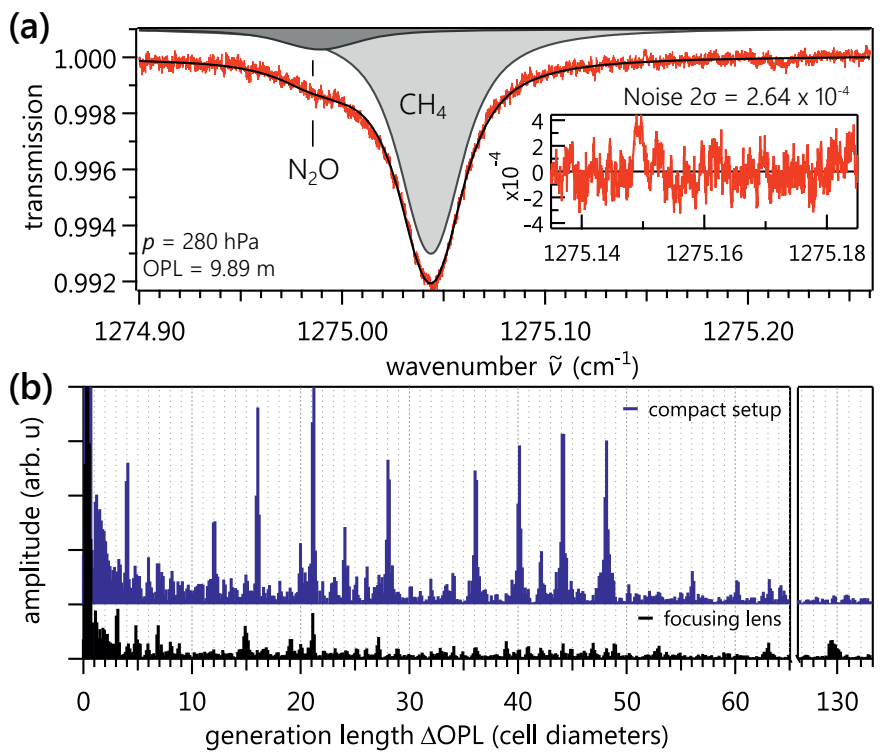

Fig. 4. Measured transmission spectrum of $\mathrm{CH}_{4}$ (1.92 ppmv) and $\mathrm{N}_{2} \mathrm{O}$ (323 ppbv) in dry atmospheric air at $280 \mathrm{hPa}$. The inset shows the local noise on the baseline. The optical path length of $9.89 \mathrm{~m}$ is achieved by 64 passes through the SC-MPC prototype. (b) A Fourier transform of the spectral noise signal displays the occurring fringe frequencies as a function of their generation length. While the bottom trace is calculated from the data set in (a), the top trace is obtained from a spectrum acquired without the use of an additional focusing element after the cell.

However, best noise levels were obtained by adding a $\mathrm{CaF}_{2}$ lens $(f=20 \mathrm{~mm})$ in front of the MCT-detector (Vigo Systems, Poland), as depicted in Fig. 3. Intermittent continuous-wave (iCW) laser driving [13] and data acquisition was realized by in-house developed FPGA-based hardware [14]. Spectra were recorded at $1 \mathrm{kHz}$ and averaged to a $1 \mathrm{~Hz}$ data stream.

For spectroscopic characterization, we selected a strong and isolated ro-vibrational transition of methane $\left(\mathrm{CH}_{4}\right)$ at $1275.04 \mathrm{~cm}^{-1}$. Fig. 4(a) shows the recorded low pressure $(p=$ $280 \mathrm{hPa}$ ) transmission spectrum of dry atmospheric air, containing $1.92 \mathrm{ppm} \mathrm{CH}_{4}$. The absorption lines are fitted by a Voigtprofile considering the parameters from the molecular database [15]. The very low normalized noise level of $2 \sigma=2.64 \times 10^{-4}$ at an averaging time of $1 \mathrm{~s}$ even makes the $\mathrm{N}_{2} \mathrm{O}$-transitions at $1274.99 \mathrm{~cm}^{-1}$ clearly observable. Measuring $\mathrm{CH}_{4}$ at ambient pressure, we achieved sub-ppb precision after $1 \mathrm{~s}$, corresponding to an absorption-noise of $2 \times 10^{-6}$. This noise level is similar to state-of-the-art values reported for Herriott-type systems [16]. It should be noted that we obtained these results without applying fringe-suppression measures such as piezo-modulation of optical components.

Whilst an excellent noise performance is achieved, further insight about the optical behavior of the SC-MPC design is gained by the detailed investigation of the residual noise level. Besides a dark noise contribution of $2 \sigma_{\text {dark }}=1.66 \times 10^{-4}$ at $1 \mathrm{~Hz}$, the existence of optical interference fringes is revealed. These fringes are caused by beam fractions that travel different distances with respect to the main beam. In air, the fringe generating length, i.e. the path length difference $\triangle O P L$ between two interfering beam fractions, is related to the spectral fringe period by $\Delta \tilde{v}=\frac{1}{\triangle O P L}$, 
in $\mathrm{cm}^{-1}$. Therefore, as shown in Fig. 4(b), the individual fringe frequencies found by Fourier transforming the spectral noise signal can be displayed as a function of $\triangle O P L$ [17]. Along these lines, the peak located somewhat above $2 \times$ OPL (at 130 passes) denotes classical Fabry-Pérot-etaloning between the laser and detector. In contrast, noise frequency components generated at distances shorter than $(p-2)$ passes can only originate from interference by stray or scattered light. Diffraction measurements on the diamond-turned surface indicate minor surface structures of $7.9 \mu \mathrm{m}$ periodicity in axial direction. This surface imperfection is a possible source of stray light within the cell. However, improper alignment or strong deviations from Gaussian beam shapes can also lead to undesired beam-branching. For instance, by deflection at the input aperture, some radiation can reach the detector already after one pass. The interference of such a beam-portion with the main beam after a full round-trip gives rise to a peak at 63 passes [see Fig. 4(b), bottom trace]. The fringe frequency spectrum is sensitively dependent on alignment changes, suggesting that the stray light generally follows much more complex traces within the cell. Additional information about the origin of stray light is obtained by using the SC-MPC without a focusing lens. The approximately four times higher normalized noise level contains dominant frequencies at integer multiples of the cell diameter, as shown in the top curve of Fig. 4(b). The increased noise level can be explained by the focusing lens that spatially separates different directional components at its focal plane, thereby acting as a filter for undesired alternative passes. Additionally, interference-noise is induced by reflections at optical components situated after the cell that fall back into the MPC (e.g. from detector chip or window). A focusing element projects such reflections back onto the last segment, proceeding backwards and eventually creating classical etaloning. Indeed, the absence of a lens leads to a clear attenuation of the $2 \times$ OPL-frequency while the now undirected back-reflections generate numerous alternative passes. Therefore, the appropriate positioning of post-cell optical components is important - anti-reflection coatings, which were not used in our setup, are expected to further improve the noise performance.

Hence, especially if back-reflections are rigorously suppressed, the incorporation of even more segments should be possible. This might be realized in a variety of creative progressions beyond the herein proposed configuration. Most relevantly, by specific tilting of particular segments, multiple round-trip patterns can be combined within the same cell. Further options are the stacking of multiple SC-MPCs or the off-plane beam coupling to create two layers of axially offset reflections, which allows a denser arrangement of segments. Consequently, SCMPCs can successfully compete with established solutions, also with respect to the maximal OPL.

In summary, we have presented a novel type of multipass cell that combines crucial requirements for mobile and mass-critical spectrometers. The monolithic circular arrangement of concave mirror segments ensures optically and mechanically stable beam folding, significantly improving the interference noise performance. This design renders pre-cell beam shaping obsolete, giving rise to very compact setups and minimal optical path length outside the sampling volume. High versatility is provided by the freedom to adjust the optical path length and the cell diameter according to individual constraints, while the dimensioning follows simple mathematical relations. We have demonstrated very low noise levels in a direct absorption spectroscopic setup and the possibility of high precision methane-measurements at ambient conditions. The segmented circular multipass cell (SC-MPC) is thereby confirmed as a suitable tool for a variety of future applications in compact and lightweight absorption spectroscopy.

\section{FUNDING INFORMATION}

This work is supported by the Swiss National Science Foundation (SNSF) 157208.

\section{ACKNOWLEDGMENTS}

The authors wish to acknowledge the help of Herbert Looser and Chang Liu (DAQ-system), Philipp Scheidegger (electronics), Urs Hintermüller (construction), Badrudin Stanicki (discussion), and LT Ultra-Precision $\mathrm{GmbH}$.

\section{REFERENCES}

1. R. F. Curl, F. Capasso, C. Gmachl, A. A. Kosterev, B. McManus, R. Lewicki, M. Pusharsky, G. Wysocki, and F. K. Tittel, Chem. Phys. Lett. 487, 1 (2010).

2. T. F. Villa, F. Gonzalez, B. Miljievic, Z. D. Ristovski, and L. Morawska, Sensors. 16, 1072 (2016).

3. D. Richter, P. Weibring, J. G. Walega, A. Fried, S. M. Spuler, and M. S. Taubman, Appl. Phys. B 119, 119 (2015).

4. W. Gurlit, R. Zimmermann, C. Giesemann, T. Fernholz, V. Ebert, J. Wolfrum, U. Platt, and J. P. Burrows, Appl. Opt. 44, 91 (2005).

5. C. R. Webster and P. R. Mahaffy, Planet. Space Sci. 59, 271 (2011).

6. J. B. McManus, P. L. Kebabian, and M. S. Zahniser, Appl. Opt. 34, 3336 (1995).

7. B. Tuzson, M. Mangold, H. Looser, A. Manninen, and L. Emmenegger, Opt. Lett. 38, 257 (2013).

8. M. L. Thoma, R. Kaschow, and F. J. Hindelang, Shock. Waves 4, 51 (1994).

9. H. Kogelnik and T. Li, IEEE J. Proc. 54, 1312 (1966).

10. M. Mangold, B. Tuzson, M. Hundt, J. Jágerská, H. Looser, and L. Emmenegger, J. Opt. Soc. Am. A 33, 913 (2016).

11. M. Graf, H. Looser, L. Emmenegger, and B. Tuzson, Opt. Lett. 42, 3137 (2017).

12. A. E. Siegman, Lasers (University Science Books, 1986).

13. M. Fischer, B. Tuzson, A. Hugi, R. Brönnimann, A. Kunz, S. Blaser, M. Rochat, O. Landry, A. Müller, and L. Emmenegger, Opt. Express 22, 7014 (2014).

14. C. Liu, B. Tuzson, P. Scheidegger, H. Looser, B. Bereiter, M. Graf, M. Hundt, O. Aseev, D. Maas, and L. Emmenegger, Rev. Sci. Instrum. (2018). (to be published).

15. L. S. Rothman et al., J. Quant. Spectrosc. Radiat. Transf. 130, 4 (2013). HITRAN2012 special issue.

16. J. B. McManus, M. S. Zahniser, D. D. Nelson, J. H. Shorter, S. C. Herndon, E. C. Wood, and R. Wehr, Opt. Eng. 49 (2010).

17. J. B. McManus, M. S. Zahniser, and D. D. Nelson, Appl. Opt. 50, A74 (2011). 